\title{
Information Transfer through Online Summarizing and Translation Technology
}

\author{
Sanja Seljan*, Ksenija Klasnić**, \\ Mara Stojanac*, Barbara Pešorda*, Nives Mikelić Preradović* \\ *Department of Information and Communication Sciences, \\ **Department of Sociology \\ Faculty of Humanities and Social Sciences, University of Zagreb \\ Ivana Lučića 3, Zagreb, Croatia \\ sseljan@ffzg.hr, kklasnic@ffzg.hr, \\ mstojana@ffzg.hr, bpesorda@ffzg.hr,nmikelic@ffzg.hr
}

\section{Summary}

Information access - presented in proper language, in understandable way, at the right time and right place can be of considerable importance. Information and communication technology, wrapping also human language technologies, can play important role in information transfer to the specific user. Translation technology along with summarizing technology has opened new possibilities and perspectives, requiring in the same time the critical opinion in information analysis. The main purpose of this research is to present the impact of text summarization and online machine translation tools on information transfer.

The research was performed on texts taken from online newspapers in five domains (politics, news, sport, film and gastronomy) in English, German and Russian languages. The total of $N=240$ evaluations were analysed, performed by the same three evaluators.

In the research three types of assignments were made. The first assignment was to evaluate machine-translated sentences at the sentence level for the three language pairs (English-Croatian, German-Croatian and Russian-Croatan). In the second task, the similar evaluation was performed, but at the whole text level. In the third assignment, which was related to information transfer, the evaluators were asked to evaluate the overall quality of the texts process in the pipelined process (online summarization and online machine translation) for English and German. Assessment was based on the finding the answers to the following questions - who, what, when, where, and how? The results were analysed by ANOVA, t-test and binary logistic regression.

Keywords: information transfer, text summarization, online translation tools, evaluation, statistical analysis, ANOVA, t-test 


\section{Introduction}

Information access - presented in proper language, in understandable way, at the right time and right place can be of crucial importance. Information and communication technology, wrapping also human language technologies, can play important role in information transfer to the specific user. One of tasks stated in Tongia et al. (2005) was to make ICT universally available, accessible, and affordable, usable and applicable.

In the situation when acquiring up-to-date information is important, automatic processes and human language technologies can play an important role. Huge amount of data cannot be managed and analysed by humans. Moreover, access is often limited, presented in various forms, of different length and in various languages, often not easily retrieved. That is the situation where automatic or semi-automatic processes of summarization and machine translation can provide the basic insight. Being piled up with huge amount of data, users have to discern valid from invalid information and to decide whether to read or not the whole documents. Because of growth of non-English speakers, large amount of information is often needed on target languages. Therefore, information access, cross-language information retrieval and information transfer represent one step further in global communication.

There are various projects dealing with information access and information retrieval, including human language technologies including online summarization and machine translation. But those free online tools are mainly created for widely spoken languages, while for less-resourced languages (e.g. Croatian) they exist mainly as standalone applications. Online summarization tool for Croatian - CroWebSum was presented by Mikelic Preradovic et al. (2010) yet not integrated in the broader process for information transfer. Free online machine translation services for Croatian still obtain lower grades than for other languages (Seljan et al. 2015a, 2015b), due to scare language resources, differences between language pairs, specific domains, etc. and depending on user's expectations.

In the paper, the pilot research was performed in order to evaluate information transfer by use of free online summarization tool and by two online machine translation tools. Information transfer was evaluated by questions: who, what, when, where, and how? Information quality, completeness, understanding and, finally, reader's possibility to recognize information was analysed. This pilot research was made in five different domains: politics, news, sport, film and gastronomy. All texts were firstly summarized and then machine translated from English, German and Russian into Croatian language. Evaluation was performed by three different evaluators, using t-test between two types of evaluations and between the two systems. The main limitation of the pilot study was small number of summaries included in the pilot research. 


\section{Related work}

Summarization and machine translation tools are often integrated as modules into various systems dealing with information access or cross-language information retrieval. An example of automatic public service is Europe Media Monitor (EMM) developed by Joint Research Centre (JRC), Directorate General of the European commission performing real-time monitoring and analysis in order to detect threats (natural disasters, diseases, etc.). The system is developed for more than 60 languages, performing gathering, clustering, information extraction, summarisation, machine translation and generation (Steinberger et al., 2009).

The paper presented by Damianos et al. (2003) describes the MiTAP system using human language technologies created for monitoring of infectious disease outbreaks providing multilingual information access to various resources in 8 languages. MITRE is the system of Text and Audio Processing attempting to solve these problems using natural language technology and careful focus on the end user. The system is designed to perform, among other, machine translation and summarization.

Afantenos et al. (2005) bring survey on summarization of medical documents used in order to quickly determine the main points of a document, elaborating on types of summaries, input factors (single or multi-document, languages, text, speech or multimedia), purpose factors (informative or indicative summaries, generic or user-oriented, general or domain-specific), output factors (quality, extracts or abstracts), evaluation methods (intrinsic or extrinsic) and various summarization techniques. It focuses especially on summarization in medical domain. Possible evaluations could be based on utility values, relevance, information inclusion, reading comprehension, or other.

Chin-Yew (1999) describes the design and implementation of MuST - a multilingual information retrieval, summarization and translation system which enables to perform cross-language information retrieval, summarization and machine translation.

Xiaojun et al. (2010) present cross-language document summarization aiming to produce summary in a target language, focusing on English-Chinese, with purpose to understand the major content. The future plans include manual translation of reference summaries and automatic ROUGE metric to perform automatic evaluation.

Chieze et al. (2010) present information system created for legal professionals which integrates natural language processing technologies, like text classification and summarization. Text is then submitted for bidirectional statistical machine translation (SMT) between English and French. 


\section{Online text summarization tools}

Text summaries represent a necessity in today's information age. Due to huge savings in time, they represent a useful tool for managing the vast available online texts. The process of summarization reduces the complexity and length of the original document, providing the visibility of the subject matter and key ideas of the work. (Mikelic Preradovic, Vlainic, 2013)

„Text summarization represents a method of extracting relevant portions of the input document, presenting the main ideas of the original text. It is a process of condensing a source document into its shorter version preserving the information content."(Mikelic Preradovic, Vlainic, 2013) Automatic text summarizations are being used for summarizing news to SMS or WAP-format, for mobile phones of PDAs or for TTS systems. It is also used for compressed descriptions of the search results in the search engines as well as in keyword directed subscriptions of news which are summarized and sent to the user. Summaries are useful for orientation (google maps) and in a decision making process (e.g. TV guide).

There are various summarization systems which use statistical or linguistic approach or the combination of these two. Although these summarization systems mainly rely ,on the shallow features of the text, they all generate informative extracts satisfying quality expectations of the human users" (Mikelic Preradovic et al., 2014, 9). There are several basic types of summaries: informative and indicative (based on the aim of the summary). Indicative summaries give the review of the summarized information from the most relevant topics in the document. Informative reduce the quantity of the information, but still keep the relevant information (Mikelic Preradovic et al, 2007). Summarization techniques can be classified into three levels (Vlainic, Mikelic Preradovic, 2013):

- surface methods - relying on the frequency of words, sentence position, words in the title or to the presence of cue phrases in text

- entity level - model text entities and the semantic relationships between these entities; the relations between entities are based on similarity, proximity and cohesion

- discourse level methods - model the document's global structure and its relation to the communicative goals, taking into account the rhetorical structure of the text.

The summarized text should give the answers to the following questions: who, what, when, where, and how? This pilot research will present the extent to which the information obtained by text summarization changed the quality, completeness and the original meaning of the text which is important while evaluating the quality of content. Attempts will be made in order to evaluate the final information transfer after using the results of three respondents who gave answers to the mentioned questions of the text which was created after the summarizing and translating. 


\section{Online translation tools}

In recent years, machine translation technology develops to the extent that it causes great interest in every possible area. In the education market, the international institutions and many other places, machine translation technology is searching for the opportunity and place for its integration. Machine translation makes quick and easy translation from one natural language into another. The aim of this paper is to show the impact of online machine translation tools to information transfer. Certain online machine translation tools will show the quality of translation and whether the information is lost or not. Summarized text was machine-translated by use of freely available online translation technology. In the research the role of both technologies is analysed in the process of conveying and understanding text from one natural language into another - in this case, from English, German and Russian into Croatian language by use of two types of technology - well-known Google Translate and Yandex Translate.

Nowadays when almost everyone can produce and share various content at the Internet, it is highly important to know sources and tools that are of good quality, precision and accuracy. However, the question is how can one say with certainty that one automatic translation system is more "suitable" or "better" than the other? Evaluation of machine translation can answer this question, but it is a complicated task due to difficulty and complexity of the process itself. Human and automatic evaluations are two types of evaluation of machine translation. As the human evaluation requires more costs and time, automatic evaluation is requisite in the process of machine translation.

Evaluation of machine translated text for Croatian, still obtain lower scores by various online tools when using different methods -1 ) human evaluation with one or more reference translations or 2) various automatic metrics (Seljan, 2015a; Seljan and Dunđer, 2015b).

In this paper, human evaluation was used. When texts from five different categories of each language were translated, respondents were given the text for evaluation. The respondents needed to perform quality evaluation of translated text comparing sentence by sentence individually in the target and source language, after the text was firstly summarized. Then they evaluated the quality of the whole text after it was only translated by each tool, not having to compare it individually sentence by sentence as they did in the previous assignment.

\section{Research Methodology}

The research has been conducted in order to receive required conclusions about information transfer by dint of questions posed to three respondents, native Croatian speakers. The corpus that was used in this research contained texts from three different languages - English, German and Russian. Each language comprised texts from five different categories: 
- politics,

- news,

- sport,

- film and

- gastronomy.

In the evaluation process, the same three native Croatians speakers participated. The total of $\mathrm{N}=240$ evaluations were analysed:

In the first task 90 evaluations for machine translated texts were made (3 language pairs, 5 domains, 3 evaluators, 2 online tools) where 60 evaluations for the pipelined process of summarization and machine translation (2 language pairs, 5 domains, 3 evaluators, 2 online tools) and 30 evaluations for the machine-translation process ( 1 language pair, 5 domains, 3 evaluators, 2 online tools). This evaluation was made at the sentence level.

In the second task, the similar evaluation was performed, but at the whole text level: 90 evaluations (3 languages, 5 domains, 3 evaluators, 2 online tools).

In the third task, which was related to information transfer, 60 evaluations were done (2 languages, 5 domains, 3 evaluators, 2 pipelined processes)

Each evaluator had three assignments.

The first assignment was to evaluate machine-translated sentences at the sentence level for the three language pairs (English-Croatian, German-Croatian and Russian-Croatan). Machine translation was made by two online services: Google Translate and Yandex Translate for all three languages. Texts on English and German were firstly summarized and then machine translated into Croatian language. The summarization was made from 108 sentences to 47 sentences in English and from 103 sentences into 49 sentences for German. As the summarization for Russian language was not possible, evaluation of text accuracy for Russian-Croatian was made only on the basis of machine translation services, without summarization step. Texts on English and German were previously summarized by online summarization tool Swesum. on the scale from 1 to 5 to each sentence (partial values as 1.0, 1.5, 2.0 etc. were also allowed in the evaluating process) using the following scale: $1=$ not understandable, not enough information, $2=$ hardly understandable, necessary to repair almost everything, $3=$ understandable, but many changes needed, $4=$ very good, but with some mistakes, $5=$ excellent. The evaluators could compare the sentences from the source and target languages at the same time. The number of sentences depended on the extent of the text. Therefore data analysis was performed on the basis of average score of all sentences for the specific domain.

The second assignment consisted in quality evaluation of the translated text as a whole. Moreover, the respondents did not have the text from the source language to compare it to the translated text. Also, likewise in the first assignment, they gave quality score to the text ranging from 1 to 5 . 
In the third assignment, which was related to information transfer, the respondents were asked to evaluate the overall quality of the texts process in the pipelined process (online summarization and online machine translation) for English and German. Assessment was based on the finding the answers to the following questions - who, what, when, where, and how? If the respondents coull give the answer to the asked question, they gave score 1, and if they could not give the answer, they marked 0 as an answer. Furthermore, the respondents did not have the source language to compare with.

\section{Results}

\section{Description}

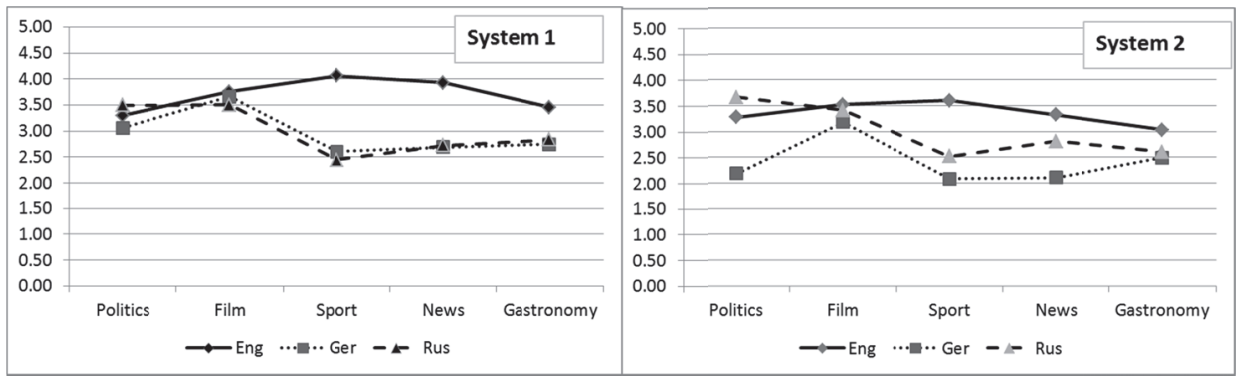

Figures 1,2. MT system 1 and system 2 evaluation - mean accuracy scores

Diagrams 1 and 2 present mean scores of machine-translated texts in five domains. Both systems obtained the best grades for the English language. The system 1 (Google Translate) obtained the second best grades for the GermanCroatian language pair and the system 2 (Yandex Translate) obtained the second best grades for the Russian-Croatian. The system 1 received close scores for the domains of film and politics for all three languages, while for other domains differences were more considerable. The system 2 received similar scores for the domain of film and gastronomy, while the politics was best represented for Russian language. The second system obtained generally better results for the Russian language as the second language and the first system for the German language as the second language.

\section{Evaluation at the sentence level}

The first assignment was to evaluate the accuracy of each sentence wherein evaluators could compare the sentences from the source and target languages at the same time.

A one-way between subjects ANOVA was conducted to compare the average level of accuracy of transmitted information when using two different free online tools for machine translation (Google Translate and Yandex Translate) 
for texts translated from English, German and Russian into Croatian at the sentence level. Normal distribution of accuracy variable across all three languages and both services was verified by Kolmogorov-Smirnov test ( $p=.057$ ).

There was a significant effect of using different online tools for different languages in accuracy of transmitted information at the $\mathrm{p}<.05$ level for the six conditions $\left[\mathrm{F}_{(5,84)}=4.78, \mathrm{p}=.001\right]$.

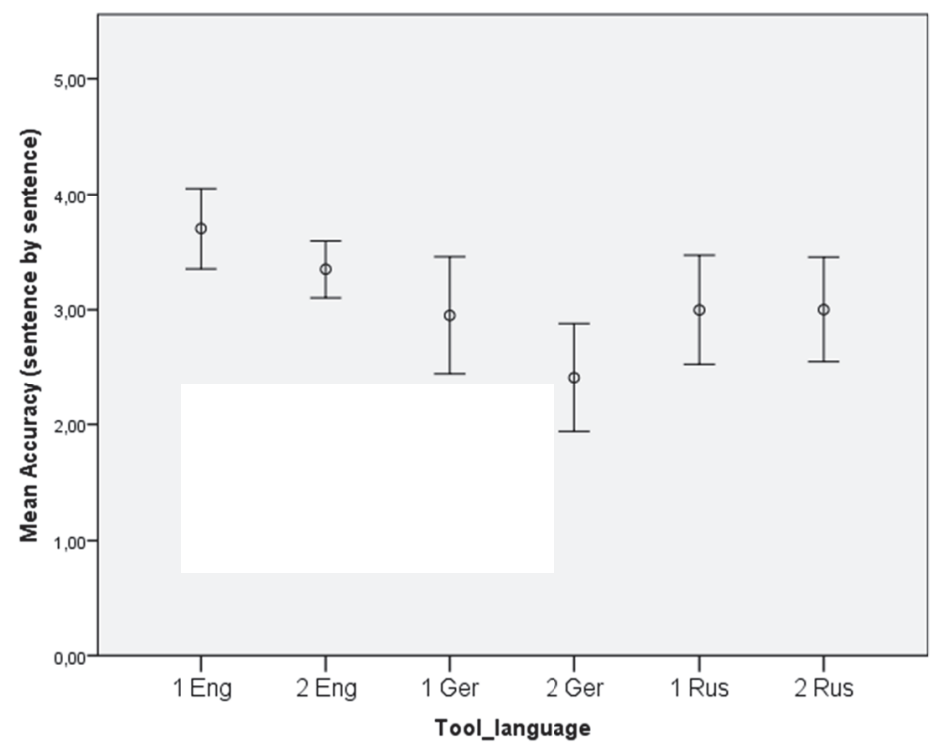

Figure 3. Error bars (mean and 95\% CI for means): accuracy by tool (1-Google Translate, 2-Yandex Translate) and language at sentence level

Post hoc comparisons using the Tukey HSD test indicated that there was no statistically significant difference among tools compared by the same language pair (e.g. English-Croatian for both tools) when transmitting information. However, two statistically significant differences in average accuracy scores were determined.

There is a difference between the use of pipelined online tools for summarization and machine translation by Google Translate from English to Croatian $(\mathrm{M}=3.70, \mathrm{SD}=0.64)$ and pipelined tools for summarization and online Yandex Translate from German to Croatian $(\mathrm{M}=2.41, \mathrm{SD}=0.85)$. Google Translate from English to Croatian resulted in higher mean accuracy than Yandex Translate from German to Croatian $(\mathrm{p}<.001)$.

Second statistically significant difference is a difference in mean accuracy scores of information between Yandex Translate from English to Croatian $(\mathrm{M}=3.35, \mathrm{SD}=0.44)$ and Yandex Translate from German to Croatian $(\mathrm{M}=2.41$, $\mathrm{SD}=0.85)$. Yandex Translate from English to Croatian resulted in higher mean accuracy than Yandex Translate from German to Croatian $(\mathrm{p}<.001)$. 


\section{Evaluation at the text level}

The second assignment consisted in quality evaluation of the translated text as a whole in which respondents did not have the text from the source language to compare it to the translated text. This task is similar to the previous one, but the evaluation is performed at the whole text level.

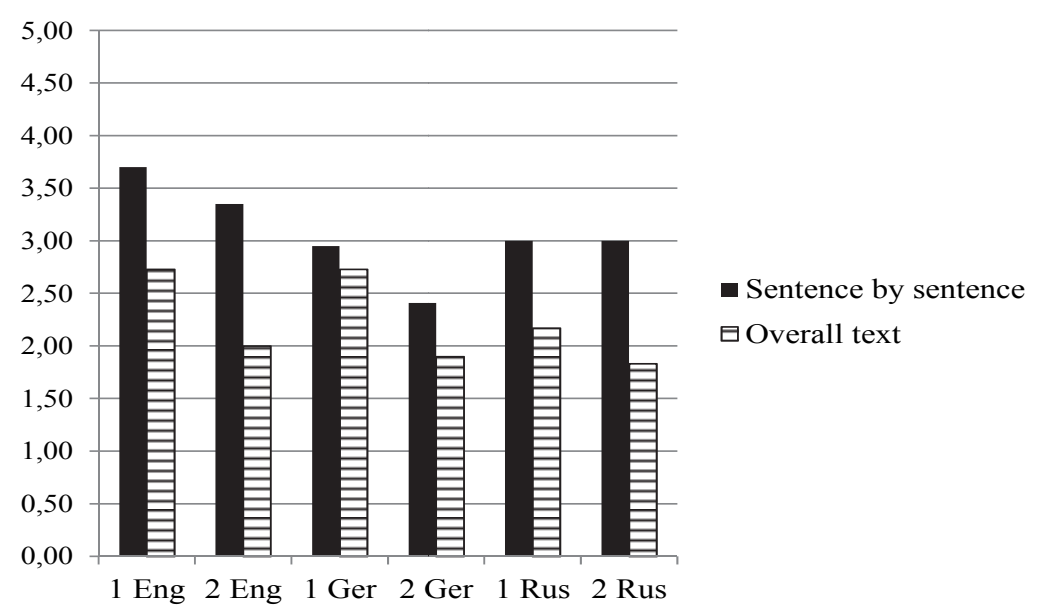

Figure 4. Comparison of sentence by sentence mean scores and text evaluation mean scores(1Google Translate, 2-Yandex Translate)

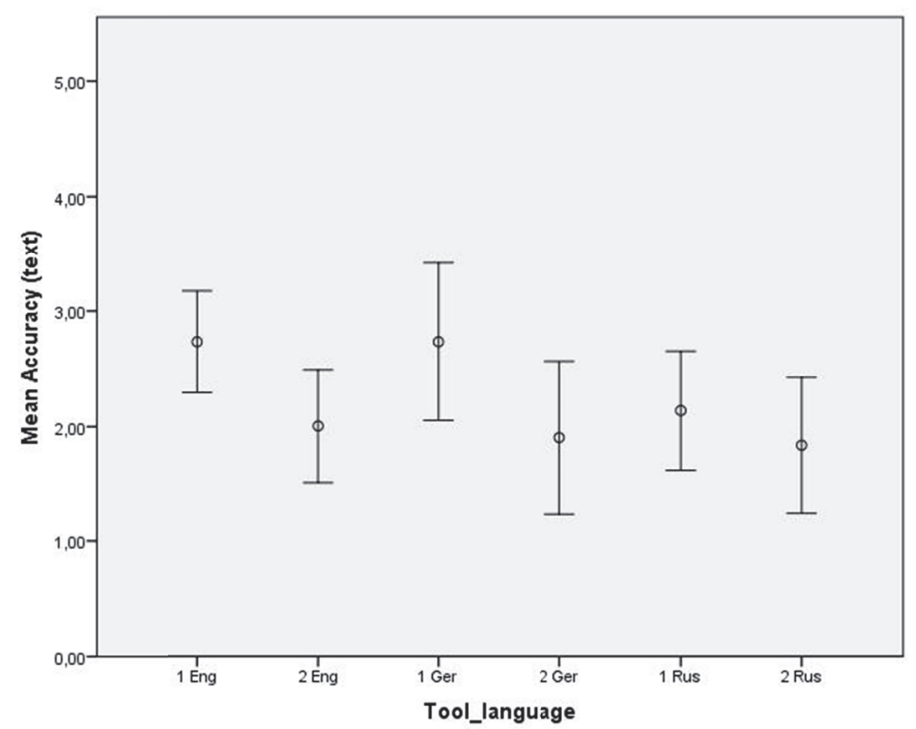

Figure 5. Error bars (mean and 95\% CI for means): accuracy by tool and language on text 
When comparing sentence by sentence translation and the overall evaluation, sentence by sentence evaluation received overall mean score of $3.07(\mathrm{SD}=.85)$, while overall evaluation received $2.22(\mathrm{SD}=1.07)$ score. This difference was statistically significant $\left[\mathrm{t}_{(89)}=7.20, \mathrm{p}<.001\right]$.

Reason for this probably lied in the fact that respondents did not have the text from the source language in order to make a comparison like they could in the first assignment. Furthermore, respondents explained that in this assignment they did not have to be as concentrated as in the previous task which was detailed due to working on each sentence individually, and not with the whole text like it was the case here.

A one-way between subjects ANOVA was conducted to compare the average level of accuracy of transmitted information when using two different free online tools for machine translation (Google Translate and Yandex Translate) for texts translated from English, German and Russian into Croatian on overall text level.

There was a significant effect of using different online tools for different languages in accuracy of transmitted information at the $\mathrm{p}<.05$ level for the six conditions $[\mathrm{F}(5,84)=2.35, \mathrm{p}=.048]$.

Post hoc comparisons using the LSD test indicated that there was one statistically significant difference among tools compared by the same language and three statistically significant differences among tools compared by different languages.

The only statistically significant difference among tools compared by the same language is for German language, between the use of machine translation by Google Translate from German to Croatian $(\mathrm{M}=2.73, \mathrm{SD}=1.24)$ and online machine translation Yandex Translate from German to Croatian $(\mathrm{M}=1.90$, $\mathrm{SD}=1.20)$. Google Translate from German to Croatian resulted in higher mean accuracy than Yandex Translate from German to Croatian $(\mathrm{p}=.030)$.

Second statistically significant difference is a difference in mean accuracy scores of information transfer between Google Translate from English to Croatian $(\mathrm{M}=2.73, \mathrm{SD}=0.80)$ and Yandex Translate from German to Croatian $(\mathrm{M}=1.90, \mathrm{SD}=1.20)$. Google Translate from English to Croatian resulted in higher mean accuracy than Yandex Translate from German to Croatian $(\mathrm{p}=.030)$.

Third statistically significant difference is a difference in mean accuracy scores of information between Google Translate from English to Croatian ( $\mathrm{M}=2.73$, $\mathrm{SD}=0.80)$ and Yandex Translate from Russian to Croatian $(\mathrm{M}=1.83, \mathrm{SD}=1.06)$. Google Translate from English to Croatian resulted in higher mean accuracy than Yandex Translate from Russian to Croatian ( $\mathrm{p}=.019)$.

Fourth statistically significant difference is a difference in mean accuracy scores of information transfer between Google Translate from German to Croatian $(\mathrm{M}=2.73, \mathrm{SD}=1.23)$ and Yandex Translate from Russian to Croatian $(\mathrm{M}=1.83$, 
$\mathrm{SD}=1.06)$. Google Translate from German to Croatian resulted in higher mean accuracy than Yandex Translate from Russian to Croatian $(\mathrm{p}=.019)$.

\section{Information transfer}

In the last assignment the respondents were asked to evaluate the overall quality of the texts based on the finding the answers to the following questions - who, what, when, where, and how?

The average information score for German was 3.8 and for English 4.4. English summaries missed mainly where? and when? information. German summaries also answered who? and what? questions, more when? information, but missed more where? information. The best score was obtained for who? (0.95), followed by what? (0.87), how? (0.83), where? (0.72) and when? (0.60).

Binary logistic regression analyses was used to test whether accuracy evaluations for English-Croatian and German-Croatian translations of both systems can predict the odds of giving the answers to five listed questions. This analysis was performed using sentence level because of higher accuracy scores.

Four out of five analyses (dependent variables: who?, what?, where? and when?) did not show any statistical significance of the effect of accuracy. Accuracy has shown to be statistically significant predictor only for the odds of giving the answers to how? question. Analysis showed that for a one-unit increase in accuracy on sentence by sentence level the odds of giving the answer to the question how? for transmitted information increases 6.3 times (95\% C.I.: 2.1 18.5) $(\mathrm{p}=.001)$.

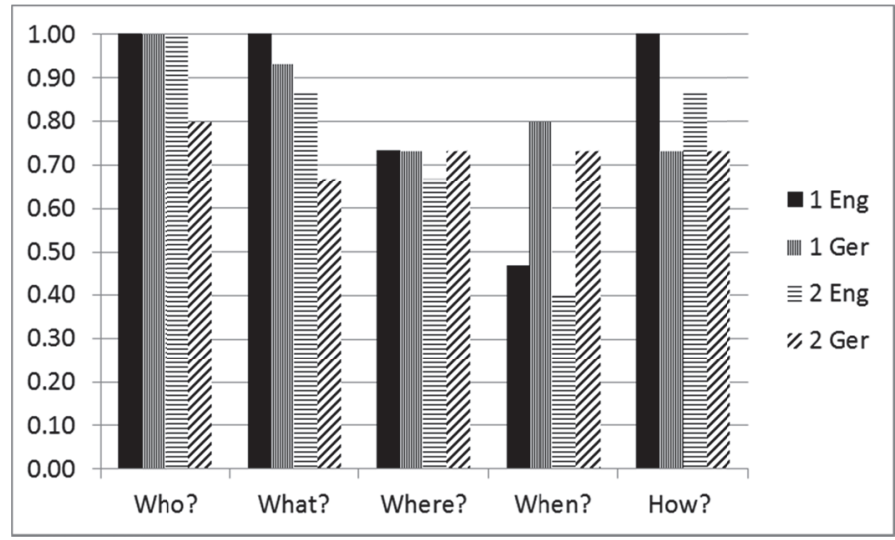

Figure 6. Information transfer in summaries across all domains 


\section{Conclusion}

The paper presents information transfer in five domains (politics, news, sport, film and gastronomy) for text taken from online newspapers for 3 languages (English, German and Russian). In the research three types of assignments were made.

The first assignment was to evaluate machine-translated sentences at the sentence level for the three language pairs (English-Croatian, German-Croatian and Russian-Croatan). A one-way between subjects ANOVA was conducted to compare the average level of accuracy of transmitted information when using two different free online tools for machine translation (Google Translate and Yandex Translate).

In both systems, the best grade was obtained for the English language. The second best grades for Google Translate were obtained for the German-Croatian language pair and for the Yandex Translate it was Russian-Croatian. Overall scores for the German-Croatian were better for Google Translate, and for Russian-Croatian the overall scores were better, but not statistically significant, for Yandex Translate.

When comparing different language pairs and different tools, there was a significant effect in accuracy of transmitted information at the $\mathrm{p}<.05$ level for the six conditions $\left[\mathrm{F}_{(5,84)}=4.78, \mathrm{p}=.001\right]$.

Post hoc comparisons using the Tukey HSD test indicated that there was no statistically significant difference among tools compared by the same language pair (e.g. English-Croatian for both tools) when transmitting information. However, two statistically significant differences in average accuracy scores were determined: between machine-translated texts by Google Translate for EnglishCroatian $(\mathrm{M}=3.70, \mathrm{SD}=0.64)$ and Yandex Translate for German-Croatian $(\mathrm{M}=2.41, \mathrm{SD}=0.85)$. Google Translate from English to Croatian resulted in higher mean accuracy than Yandex Translate from German to Croatian $(\mathrm{p}<.001)$.

Second statistically significant difference is a difference in mean accuracy scores between Yandex Translate for English-Croatian $(\mathrm{M}=3.35, \mathrm{SD}=0.44)$ and Yandex Translate for German-Croatian $(\mathrm{M}=2.41, \mathrm{SD}=0.85)$. Yandex Translate from English to Croatian resulted in higher mean accuracy than Yandex Translate from German to Croatian ( $\mathrm{p}<.001)$.

The second assignment consisted in quality evaluation of the whole translated text, without possibility to compare with source language text.

When comparing sentence by sentence translation and the overall evaluation, sentence by sentence evaluation received overall mean score of $3.07(\mathrm{SD}=.85)$, while overall evaluation received $2.22(\mathrm{SD}=1.07)$ score. This difference was statistically significant $\left[\mathrm{t}_{(89)}=7.20, \mathrm{p}<.001\right]$.

A one-way between subjects ANOVA was conducted to compare the average level of accuracy of transmitted information when using two different free 
online tools for machine translation for texts translated from English, German and Russian into Croatian on overall text level.

There was a significant effect of using different online tools for different languages in accuracy of transmitted information at the $\mathrm{p}<.05$ level for the six conditions $[F(5,84)=2.35, p=.048]$.

The only statistically significant difference among tools compared by the same language is for German language, between the use of machine translation by Google Translate from German to Croatian $(\mathrm{M}=2.73, \mathrm{SD}=1.24)$ and online machine translation Yandex Translate from German to Croatian $(\mathrm{M}=1.90$, $\mathrm{SD}=1.20)$. Google Translate from German to Croatian resulted in higher mean accuracy than Yandex Translate from German to Croatian ( $\mathrm{p}=.030)$.

In the third assignment, which was related to information transfer, the evaluators were asked to evaluate the overall quality of the texts processed in the pipelined process (online summarization and online machine translation) for English and German. Assessment was based on the finding the answers to the following questions - who, what, when, where, and how? The average information score for German was 3.8 and for English 4.4.

Binary logistic regression analyses was used to test whether accuracy evaluations for English-Croatian and German-Croatian translations at the sentence level of both systems can predict the odds of giving the answers to five listed questions. Analysis showed that for a one-unit increase in accuracy on sentence by sentence level the odds of giving the answer to the question how? for transmitted information increases 6.3 times (95\% C.I.: 2.1 - 18.5) ( $\mathrm{p}=.001)$.

Taken together, results suggest significant differences in information transfer when using different online tools. Although they work best for the English language, there are significant differences among other languages and online tools. The second aspect is the user information perception who gave significantly higher scores in sentence by sentence evaluation, than on the whole text evaluation. Information transfer in the pipelined process of summarization and online translation shows significant connection between accuracy and the question how? However, these results are to be taken as preliminary due to small number of test data analysed in this pilot research. The following pilot research would include finding methods for improving results for the specific language or by adding online terminology/ontology resources.

\section{References}

Afantenos, Stergos D.; Karkaletsis, Vangelis; Stamatopoulos, Panagiotis. // Summarization from Medical Documents: Survey. Journal of Artificial Intelligence in Medicine. 33(2005);157-177

Chieze, Emmanuel; Farzindar, Atefeh; Lapalme, Guy. An Automatic System for Summarization and Information Extraction of Legal Information. // Semantic Processing of Legal Texts. Lecture Notes in Computer Science. 6036 (2010); 216-234

Chin-Yew, Lin. Machine Translation for Information Access across the Language Barrier: te MuST System. Machine Translation Summit VII, 1999 
Damianos, Laurie E.; Wohlever, Steve; Kozierok, Robyn; Ponte, Jay M. MiTAP: A Case Study of Integrated Knowledge Discovery Tools. // 36th Hawaii Int. Conf. on System Sciences (HICSS36); 2003; 69

Hofman, Hans. How to keep digital records understandable and usable through time? // Proceedings Long-Term Preservation of Electronic Records / Paris, 2001

Lee, Alice Y.L.; So, Clement Y.K. Media Literacy and Informtaion Literacy: Similarities and Diferences. // Comunicar. 21 (2014), 42; 137-145

Mikelic Preradovic, Nives; Ljubesic, Nikola; Boras, Damir. Croatian web text summarizer (CroWebSum). // 32nd Int. Conf. on Information Technology Interfaces (ITI). 2010; 109-114

Mikelic Preradovic, Nives; Boras, Damir ; Vlainic, Marta. Importance of Surface Methods in Human and Automatic Text Summarization. // Journal of Computers. 8 (2014); 9-16

Tongia, R.; Subrahmanian, E.; Arunachalam, V. S. Information and Communications Technology. // Information and Communication Technology for Sustainable Development: Defining a Golobar Research Agenda. / Bangalore: Allied Publishers, 2005; 19-42

Seljan, Sanja; Tucaković, Marko; Dunđer, Ivan. Human Evaluation of Online Machine Translation Services for English/Russian-Croatian. // Advances in Intelligent Systems and Computing - New Contributions in Information Systems and Technologies. Springer. 353 (2015a); 10891098

Seljan, Sanja; Dunđer, Ivan. Machine Translation and Automatic Evaluation of English/RussianCroatian. // Proceedings of Corpus Linguistics. St. Petersburg State Univ., 2015; 72-79

Steinberger, Ralf; Pouliquen, Bruno; van der Goot, Erik.n introduction to the Europe Media Monitor family of applications. // Information Access in a Multilingual World-Proceedings of the SIGIR 2009 Workshop (SIGIR-CLIR'2009). 2009; 1-8

Vlainic, Marta; Mikelić Preradovic, Nives. Comparative Study of Automatic Text Summarization System Performance. // Recent Advances in Information Science. 13 (2013); 222-227.

Xiaojun, Wan; Huiying, Li; Jianguo, Xiao. Cross-Language Document Summarization Based on Machine Translation Quality Prediction. Proceedings of the 48th Annual Meeting of the Association for Computational Linguistics (ACL). 2010; 917-926 